DAMTP-2007-27

arXiv:0704.3728 [hep-th]

\title{
Quantum Effects in Black Holes from the Schwarzschild Black String?
}

\author{
Alessandro Fabbri ${ }^{1}$ \\ Departamento de Física Teórica and IFIC, Universidad de Valencia-CSIC, \\ C. Dr. Moliner 50, Burjassot-46100, Valencia, Spain.
}

\section{Giovanni Paolo Procopio ${ }^{2}$}

D.A.M.T.P., Centre for Mathematical Sciences, University of Cambridge, Wilberforce Road, Cambridge CB3 0WA, U.K.

\begin{abstract}
The holographic conjecture for black holes localized on a 3-brane in RandallSundrum braneworld models RS2 predicts the existence of a classical 5D time dependent solution dual to a 4D evaporating black hole. After briefly reviewing recent criticism and presenting some difficulties in the holographic interpretation of the Gregory-Laflamme instability, we simulate some basic features of such a solution by studying null geodesics of the Schwarzschild black string, in particular those propagating nontrivially in the bulk, and using holographic arguments.
\end{abstract}

\footnotetext{
1 afabbri@ific.uv.es

2 g.p.procopio@damtp.cam.ac.uk
} 


\section{Introduction}

Holography is an important tool to relate seemingly different theories living in different spacetime dimensions. The $A d S / C F T$ correspondence [1] adapted to the RandallSundrum braneworld model RS2 [2] predicts that classical bulk physics in $A d S_{5}$ is dual to a particular cut-off CFT (namely $\mathcal{N}=4 \mathrm{SU}(N) \mathrm{SYM}$ in the planar limit) coupled to gravity living on the brane. A concrete realization of this is provided by the calculation of the correction to the Newtonian potential on the brane, which can be performed in two very different ways giving the same result. Studying linear gravitational perturbations (5D gravitons) of the form $h_{\mu \nu}=e^{i p x} H_{\mu \nu}(z)$, with $p^{2}=m^{2}$, around the Randall-Sundrum vacuum

$$
d s^{2}=e^{-2 k|z|}\left[\eta_{\mu \nu} d x^{\mu} d x^{\nu}\right]+d z^{2}
$$

where $k$ is related to the five-dimensional cosmological constant by $k=\sqrt{-\Lambda_{5} / 6}$, Garriga and Tanaka [3] found that the gravitational potential on the brane $(z=0)$ generated by a mass $M$ is

$$
\phi(r)=\frac{M}{r}\left(1+\frac{2}{3} \frac{1}{k^{2} r^{2}}\right),
$$

for scales $r \gg 1 / k$. The first, Newtonian, term is given by the zero-mode $\left(m^{2}=0\right)$ bound to the brane, while the second, corrective, term is the contribution induced by the massive modes $\left(m^{2} \neq 0\right)$ living in the bulk. On the other hand, from a pure 4D perspective, Duff [4] found that the quantum corrected Newtonian potential is given by

$$
\phi(r)=\frac{M}{r}\left(1+\frac{\alpha \hbar}{r^{2}}\right),
$$

where the coefficient $\alpha=\frac{2 N^{2}}{3 \pi}$ depends on the relevant CFT, $N^{2}$ counting the number of degrees of freedom. If one combines this result with the holographic relation

$$
\frac{1}{k^{2}}=\frac{\hbar N^{2}}{\pi}
$$

one recovers exactly (2) [5] (see also [6]). The equivalence between the expressions (2) and (3) shows that the quantum corrections due to the CFT are classically given by the bulk massive modes $m^{2} \neq 0$.

Application of holographic ideas beyond the linearized level, in particular to the extreme case of black holes, has led to the conjecture that for large masses "black hole solutions localized on the brane in the $A d S_{D+1}$ braneworld which are found by solving the classical bulk equations in $A d S_{D+1}$ correspond to quantum-corrected black holes in $D$ dimensions, rather than classical ones" [7].

Evidence for this conjecture comes from the explicit solutions of black holes localized on a 2-brane in $A d S_{4}$ [8] and on a 1-brane in BTZ [9], but in the physically relevant case of a black hole on a 3-brane in $A d S_{5}$ things are much more complicated 
and no static solutions of this type have been found yet [10]. The lack of a full 5D braneworld solution giving a 4D static asymptotically flat black hole localized on a 3brane is naturally explained by the holographic conjecture: a quantum corrected 4D black hole cannot be static as it would evaporate via the Hawking effect.

Recently, as a counterexample to this argument the RS2 Schwarzschild black string solution [11]

$$
d s^{2}=e^{-2 k|z|}\left[-\left(1-\frac{2 M}{r}\right) d t^{2}+\frac{d r^{2}}{\left(1-\frac{2 M}{r}\right)}+r^{2} d \Omega^{2}\right]+d z^{2}
$$

was considered in [12]. Indeed, this solution gives the classical Schwarzschild solution on the brane. To justify the absence of quantum corrections in the dual theory, it was speculated in [12] that due to strong coupling effects the number of asymptotic degrees of freedom is drastically reduced and this would imply that the radiation vanishes at leading order (where only terms which are $O\left(\hbar N^{2}\right)$ survive).

The arguments used in [12] are based on the fact that on a sphere of radius $R$ and at large 't Hooft coupling $\lambda$ the energy separation for weakly interacting states is $\sim \frac{\lambda^{1 / 4}}{R}$ and thus the spectrum is lifted to infinite energy apart from the $O(1)$ massless states dual to the supergravity modes of the string. However, note that in the flat limit $R \rightarrow \infty$ the above mass gap disappears and this is consistent with the perturbative calculation (2), which in the dual CFT gives $O\left(N^{2}\right)$ results. The same happens in cosmology [13]. It is difficult to believe that the number of massless degrees of freedom is in general $O(1)$ except for the cases where explicit verification is possible, i.e. flat and cosmological branes. Another problem with the proposed holographic interpretation of (5) is to explain why, besides the absence of a radiative term, all the quantum corrections (say, the vacuum polarization terms) are actually suppressed. Moreover, as already pointed out in [11], the singularity at $r=0$ extends all the way from $z=0$ to $z=\infty$, making the AdS horizon singular as well. ${ }^{1}$ Therefore, such solution is likely not to have a counterpart in the dual CFT.

It has been suggested that the Gregory-Laflamme (GL) instability [14], [15] of (5) is dual to Hawking radiation in the boundary theory [16]. We will show in section 2 that a quantitative comparison of the GL brane perturbed metric with that of an evaporating 4D black hole in the near-horizon region shows some problematic points. We will then turn, in section 3, to an interesting feature of the RS2 Schwarzschild black string which we discovered by studying null geodesics and in particular those propagating nontrivially in the bulk. Indeed, in the 'geometrical optics' approximation, 5D gravitational waves (in particular, bulk massive modes) travel along such geodesics and these, in turn, might contain "seeds" of quantum effects in the dual theory. The holographic interpretation of our results, in section 4 , leads us to conjecture some crucial features of the actual 5D configuration dual to an evaporating 4D black hole. This is done in section 5. Finally, in section 6 we briefly state our conclusions.

\footnotetext{
${ }^{1}$ The authors of [12] have tried to remove this problem by considering an additional brane. This in the dual theory on the brane implies that the CFT is cut-off also in the IR.
} 


\section{GL instability and Hawking radiation}

An important property of the RS2 black string comes from the analysis of its linear gravitational perturbations which leads to the well-known Gregory-Laflamme instability. In view of the holographic black holes conjecture [7] it is natural to wonder whether such classical instability corresponds, in the boundary theory, to the quantum instability of the 4D Schwarzschild spacetime via Hawking radiation.

Considering the metric (5), it was shown by Gregory [15] that the instability for the flat black string initially discovered in [14] simply generalizes to this warped case. Perturbations of the metric

$$
g_{\mu \nu} \rightarrow g_{\mu \nu}+\delta g_{\mu \nu}
$$

where

$$
\delta g_{\mu \nu}=u_{m}(z) \hat{g}_{\mu \nu}
$$

take the following form in the $r \rightarrow 2 M$ limit

$$
\begin{aligned}
& \hat{g}^{t t} \approx\left(-\frac{1}{2}+2 M \Omega\right)\left(1-\frac{2 M}{r}\right)^{-2+2 M \Omega} e^{\Omega t} \\
& \hat{g}^{r r} \approx\left(-\frac{1}{2}+2 M \Omega\right)\left(1-\frac{2 M}{r}\right)^{2 M \Omega} e^{\Omega t} \\
& \hat{g}^{t r} \approx-\left(-\frac{1}{2}+2 M \Omega\right)\left(1-\frac{2 M}{r}\right)^{-1+2 M \Omega} e^{\Omega t},
\end{aligned}
$$

while

$$
u_{m}(z)=\mathcal{A} \mathrm{J}_{2}\left(\frac{m}{k} e^{k|z|}\right)-\mathcal{B} \mathrm{N}_{2}\left(\frac{m}{k} e^{k|z|}\right)
$$

with the coefficients $\mathcal{A}$ and $\mathcal{B}$ satisfying

$$
\mathcal{A} \mathrm{J}_{1}\left(\frac{m}{k}\right)=\mathcal{B N}_{1}\left(\frac{m}{k}\right) \text {. }
$$

The suitable time coordinate to parameterize the (future) horizon is not $t$ but $v$, the ingoing Eddington-Finkelstein null coordinate $\left(v=t+r+2 M \ln \frac{r-2 M}{2 M}\right)$. In terms of $v$ the above perturbations take the form

$$
\begin{aligned}
& \hat{g}_{t t} \approx\left(-\frac{1}{2}+2 M \Omega\right) e^{\Omega v} \\
& \hat{g}_{r r} \approx\left(-\frac{1}{2}+2 M \Omega\right)\left(1-\frac{2 M}{r}\right)^{-2} e^{\Omega v} \\
& \hat{g}_{t r} \approx\left(-\frac{1}{2}+2 M \Omega\right)\left(1-\frac{2 M}{r}\right)^{-1} e^{\Omega v} .
\end{aligned}
$$

These vanish at the past horizon $(v=-\infty)$.

With the change of coordinates $(t, r) \rightarrow(v, r)$ the perturbed metric on the brane $(z=0)$ along the future horizon takes the ingoing Vaidya form

$$
d s^{2}=-\left(1-\frac{2 m(v)}{r}\right) d v^{2}+2 d v d r+r^{2} d \Omega^{2},
$$


with mass function given by

$$
m(v)=M+M\left(-\frac{1}{2}+2 M \Omega\right) e^{\Omega v} u_{m}(0) .
$$

The numerical results show that for $M=1$ the most favored instability has $m_{0} \simeq 0.2$ and $\Omega_{0} \simeq 0.05$, while for general $M$ we have $m_{0} \rightarrow \frac{m_{0}}{M}$ and $\Omega_{0} \rightarrow \frac{\Omega_{0}}{M}$. In the large $M$ (i.e. $m \ll k$ ) limit the leading order term in $u_{m}(z)$ is

$$
u_{m}(z)=\mathcal{A}\left[\mathrm{J}_{2}\left(\frac{m}{k} e^{k|z|}\right)-\frac{\mathrm{J}_{1}\left(\frac{m}{k}\right)}{\mathrm{N}_{1}\left(\frac{m}{k}\right)} \mathrm{N}_{2}\left(\frac{m}{k} e^{k|z|}\right)\right] \sim \mathcal{A} e^{-2 k|z|},
$$

where we have used the expansions $\mathrm{J}_{\nu}(x) \sim\left(\frac{x}{2}\right)^{\nu} \frac{1}{\Gamma(\nu+1)}$ and $\mathrm{N}_{\nu}(x) \sim-\frac{\Gamma(\nu)}{\pi}\left(\frac{x}{2}\right)^{-\nu}$ valid for $x \ll 1$. Note that the value for $u_{m}(0)$ used in [3] is $\sqrt{\frac{m}{2 k}}$. Moreover, the instability exists in the range $0<m<\frac{0.45}{M}$, so the perturbed black string is approximated by

$$
d s^{2}=e^{-2 k|z|}\left[-\left(1-\frac{2 m(v)}{r}\right) d v^{2}+2 d v d r+r^{2} d \Omega^{2}\right]+d z^{2},
$$

where

$$
m(v)=M+\int_{0}^{\frac{0.45}{M}} \frac{d m}{k} M\left(-\frac{1}{2}+2 M \Omega\right) e^{\Omega v} u_{m}(0) .
$$

We should compare (19) and (20) on the brane with the metric of a four-dimensional evaporating black hole in the near horizon region, which takes the form (16) [17] with

$$
\frac{d m(v)}{d v} \sim-\frac{T_{H}^{2}}{\hbar} \sim-\frac{\hbar}{M^{2}}
$$

the emission taking place starting from some initial $v_{0}$ (due to the fact that the black hole is created from a gravitational collapse). Differentiating (20) we get

$$
\frac{d m(v)}{d v} \sim-\left(\frac{1}{k M}\right)^{3 / 2} e^{\Omega v}
$$

where we have used the fact that $m \sim 1 / M$. Neither the powers of $\hbar$ (using the holographic relation (4)) nor those of $M$ match those in (21).

Despite this negative comparison, one should not give up hopes to find a solution with the holographic behaviour (21). First, it is not clear whether the boundary conditions used in [14] correspond, in the dual theory, to a black hole created via gravitational collapse. Also, we should not exclude the possibility that the dual Hawking radiation cannot be seen from a classical linear perturbation analysis in $5 \mathrm{D}$, but needs a full nonlinear treatment. 


\section{Analysis of the Black String null geodesics}

We will now focus on a different type of analysis. The KK modes/CFT modes correspondence of eqs. (2), (3) tells us that quantum effects on the brane are induced by gravitational waves, which, in turn, propagate along null geodesics of the full 5D spacetime. Keeping this in mind, let us consider the full black string

$$
d s^{2}=e^{-2 k z}\left[-\left(1-\frac{2 M}{r}\right) d v^{2}+2 d v d r+r^{2} d \Omega^{2}\right]+d z^{2},
$$

here written in advanced Eddington-Finkelstein coordinates (in the RS2 case the spacetime is cut at $z=0$ and $e^{-2 k z} \rightarrow e^{-2 k|z|}$ ).

There are two families of null geodesics in the background (23) and this can be easily seen by considering the $z$ component of the geodesics equations

$$
\ddot{x^{\alpha}}+\Gamma_{\beta \gamma}^{\alpha} \dot{x^{\beta}} \dot{x^{\gamma}}=0
$$

which reads

$$
\ddot{z}-k \dot{z}^{2}=0,
$$

where a dot denotes a derivative with respect to the affine parameter $\lambda$. The first family is associated to the solution

$$
\dot{z}=0 \text {, }
$$

i.e.

$$
z=\text { constant }
$$

including, in particular, the null geodesics on the brane $(z=0)$ for the RS2 black string. The second, the nontrivial one, is given by

$$
\dot{z}=\frac{d z}{d \lambda}=-\frac{1}{k \lambda}
$$

which is integrated to ${ }^{2}$

$$
e^{k z}= \pm \frac{1}{k \lambda}
$$

The solution to the null geodesics equations can be obtained by considering the first integrals of motion. We have (consider motion in the equatorial plane $\theta=\pi / 2$ )

$$
e^{-2 k z}\left[-\left(1-\frac{2 M}{r}\right) \dot{v}^{2}+2 \dot{v} \dot{r}+r^{2} \dot{\varphi}^{2}\right]+\dot{z}^{2}=0
$$

and, due to the fact that $\frac{\partial}{\partial v}$ and $\frac{\partial}{\partial \varphi}$ are Killing vectors,

$$
e^{-2 k z}\left[-\left(1-\frac{2 M}{r}\right) \dot{v}+\dot{r}\right]=-E
$$

\footnotetext{
${ }^{2}$ In the case of the RS2 black string, the + sign refers to geodesics propagating from the bulk towards the brane and the - sign to those propagating from the brane towards the bulk.
} 
and

$$
e^{-2 k z} r^{2} \dot{\varphi}=L
$$

where $E$ and $L$ are constants.

In the case (25) with $z=0$ (on the brane, for the RS2 black string) and $L=0$ we have the usual Schwarzschild ingoing and outgoing radial null geodesics. For ingoing geodesics $(v=$ const) we have

$$
\dot{r}=-E, \quad \dot{v}=0 \text {. }
$$

Outgoing geodesics play a key role to determine the position of the future apparent horizon on the brane, defined as the surface where the radius of the two-sphere $r$ has zero divergence $(d r / d v=0)$. From (28) and (29) and considering $\dot{v} \neq 0$ we have

$$
\begin{aligned}
& -\left(1-\frac{2 M}{r}\right) \dot{v}+2 \dot{r}=0 \\
& -\left(1-\frac{2 M}{r}\right) \dot{v}+\dot{r}=-E,
\end{aligned}
$$

from which we get

$$
\dot{r}=E, \quad \dot{v}=\frac{2 E}{\left(1-\frac{2 M}{r}\right)}
$$

and, also,

$$
\frac{d r}{d v}=\frac{1}{2}\left(1-\frac{2 M}{r}\right) .
$$

Outgoing geodesics diverge for $r>2 M(E>0)$ and converge when $r<2 M(E<0)$. The particular outgoing geodesics that remain on the future horizon

$$
r=2 M
$$

for which

$$
\frac{d r}{d v}=0
$$

are characterized by $\dot{r}=0$, and thus

$$
E=0
$$

We will perform a similar analysis for the null geodesics of the second family (26). For $L=0$ and using (27) we get ${ }^{3}$

$$
\begin{aligned}
& -\left(1-\frac{2 M}{r}\right) \dot{v}^{2}+2 \dot{v} \dot{r}=-\frac{1}{(k \lambda)^{4}} \\
& -\left(1-\frac{2 M}{r}\right) \dot{v}+\dot{r}=-\frac{E}{(k \lambda)^{2}}
\end{aligned}
$$

\footnotetext{
${ }^{3}$ It is not difficult to generalise our analysis to $L \neq 0$.
} 
Note that due to the nontrivial motion in the bulk $(\dot{z} \neq 0)$ (39) implies $\dot{v} \neq 0$. We can then divide (39) by $\dot{v}$ and subtract (40) to get

$$
\dot{r}=\frac{E}{(k \lambda)^{2}}-\frac{1}{(k \lambda)^{4}} \frac{1}{\dot{v}},
$$

which substituted into (40) gives a quadratic equation for $\dot{v}$

$$
\left(1-\frac{2 M}{r}\right) \dot{v}^{2}-\frac{2 E}{(k \lambda)^{2}} \dot{v}+\frac{1}{(k \lambda)^{4}}=0 .
$$

This equation implies the two behaviors

$$
\dot{v}=\frac{E \pm \sqrt{E^{2}-\left(1-\frac{2 M}{r}\right)}}{\left(1-\frac{2 M}{r}\right)} \frac{1}{(k \lambda)^{2}}
$$

and, from (41),

$$
\dot{r}= \pm \frac{\sqrt{E^{2}-\left(1-\frac{2 M}{r}\right)}}{(k \lambda)^{2}} .
$$

The equations obtained correspond to (radial) timelike geodesics in a 4D Schwarzschild spacetime with an affine parameter $\nu \sim \frac{1}{k^{2} \lambda}[11]$.

The purpose of this analysis is to see whether nontrivial bulk null geodesics, presumably associated to the trajectories of the massive KK modes, can give us some hints of quantum effects in the dual theory on the brane. To understand if such "quantum effects" would modify the horizon, eqs. (36)-(38) suggest that we should focus on the special case $E=0$, where we have

$$
\dot{v}=\mp \frac{1}{\sqrt{\frac{2 M}{r}-1}} \frac{1}{(k \lambda)^{2}}
$$

and

$$
\dot{r}= \pm \frac{\sqrt{\frac{2 M}{r}-1}}{(k \lambda)^{2}} .
$$

Integration of (46) is straightforward and using (27) we get

$$
\sqrt{r(2 M-r)}+2 M \arccos \sqrt{\frac{r}{2 M}}+C= \pm \frac{e^{k z}}{k},
$$

where $C$ is the integration constant. We shall now impose the boundary condition that $r \rightarrow 2 M$ when $z \rightarrow-\infty$. $^{4}$ This implies $C=0$ and, also, that the only real solution is the one with the + sign, namely

$$
\sqrt{r(2 M-r)}+2 M \arccos \sqrt{\frac{r}{2 M}}=\frac{e^{k z}}{k} .
$$




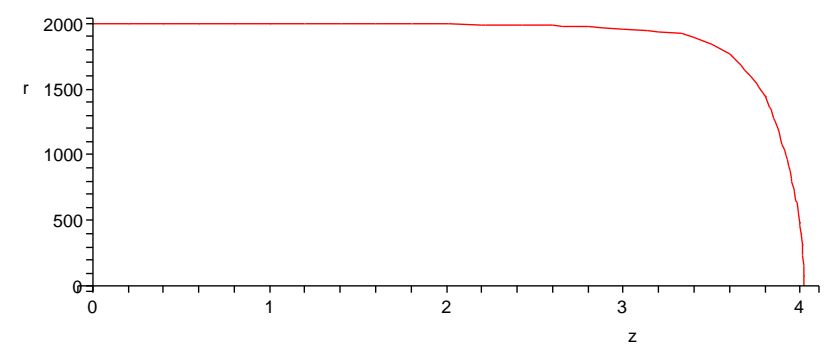

Figure 1: Plot of the radial geodesic $r$ as function of $z$ (equation (48) for $M=10^{3}$ and $k=1$.

The curve (48) is plotted for $k=1$ and $M=10^{3}$ (we are interested in the large mass regime) in fig. 1 .

From (48) in the near horizon region $r \rightarrow 2 M$ we obtain ${ }^{5}$

$$
r_{\text {bulk }}(z) \sim 2 M\left[1-\frac{e^{2 k z}}{(4 M k)^{2}}\right] .
$$

It is now interesting to evaluate $d r / d v$ in this limit, and show that is does not vanish unlike the corresponding case (37). In fact from (40) (with $E=0$ ) we get

$$
\frac{d r}{d v}=\frac{\dot{r}}{\dot{v}}=1-\frac{2 M}{r} \sim \frac{r-2 M}{2 M}
$$

and using (49),

$$
\frac{d r}{d v} \sim-\frac{1}{(4 M k)^{2}} e^{2 k z}
$$

\section{Hawking radiation in the holographic dual?}

Having established that massive modes "see" the horizon in quite a different way, compare (49) with (36) and (50) with (37), we shall now turn to the possible holographic implications of our results (see also [18]).

In the RS2 case, let us project (50) on the brane to get

$$
\frac{d r}{d v}(z=0) \sim-\frac{1}{(4 M k)^{2}}
$$

and use the holographic relation (4)

$$
\frac{d r}{d v}(z=0) \sim-\frac{\hbar N^{2}}{M^{2}} .
$$

\footnotetext{
${ }^{4}$ We will justify this choice at the end of section 5 .

${ }^{5}$ In the case $L \neq 0$ one finds $r_{\text {bulk }}(z) \sim 2 M\left[1-\frac{e^{2 k z}}{(4 M k)^{2}}\left(1+\frac{L^{2}}{4 M^{2}}\right)\right]$. The correction to the $L=0$ case is, for large masses, very small.
} 
We have seen in section 2 that for the case of evaporating black holes the apparent horizon $r^{\mathrm{AH}}=2 m(v)$ is such that, see (16) and (21),

$$
\frac{d r^{\mathrm{AH}}}{d v} \sim-\frac{\hbar}{M^{2}}
$$

The similarity with (52) is quite interesting, the only difference being that in (52) we have the multiplicative factor $N^{2}$, indicating "thermal emission" due to a large number of matter fields at the temperature $T_{\mathrm{H}} \sim \frac{\hbar}{M}$.

It is not easy to justify the holographic interpretation proposed in (52) and its comparison with (53). The black string horizon is at $r=2 M$, it is not receding. So why should bulk null geodesics in the unperturbed Schwarzschild black string know about Hawking radiation in the dual theory? We do not have a precise answer to this question. However, we note that the discovery of the Hawking effect [19] was performed in fixed background approximation, its implication being that due to the quantum corrections the Schwarzschild solution turns to a new solution with the (apparent) horizon satisfying (53). In our case, since bulk massive modes (presumably) travel along the null geodesics (26) and they are the ones responsible for the quantum effects on the brane, our result (51) makes it reasonable to expect that the black string too will be modified to a time-dependent configuration with the horizon on the dual theory on the brane evolving according to (52).

In addition to this, we know that in an evaporating black hole scenario, due to the black hole emission apparent and event horizons (coincident for the static Schwarzschild solution $r^{\mathrm{EH}}=r^{\mathrm{AH}}=2 M$ ) separate and a 'quantum ergosphere' forms in between $\left(r^{\mathrm{EH}}<r<r^{\mathrm{AH}}\right)$ [20]. Approximate calculations (valid for large $M$ ) of the location of the event horizon, when the effects of the evaporation are taken into account, show that [17, 21]

$$
r^{\mathrm{EH}}-2 M \sim-\frac{\hbar}{M}
$$

Note that the event horizon too recedes according to (53) [20]. Waves emitted from the quantum ergosphere with wavelength $\lambda_{0} \sim r^{\mathrm{AH}}-r^{\mathrm{EH}} \sim \frac{\hbar}{M}$ are detected at infinity with $\lambda_{\infty} \sim M$, which is indeed the typical size of the Hawking quanta. As in the static case $\left(r^{\mathrm{EH}}=r^{\mathrm{AH}}\right)$ there is no emission, the existence of this region is deeply connected with Hawking radiation.

In our case, (49) identifies on the brane a surface just inside the horizon, the distance from it being

$$
r_{\text {bulk }}(0)-2 M \sim-\frac{1}{M k^{2}} .
$$

Using again the holographic relation (4) we get

$$
r_{\text {bulk }}(0)-2 M \sim-\frac{\hbar N^{2}}{M}
$$

which is very similar to (54) except for the fact that the "quantum ergosphere" in the dual theory on the brane, being multiplied by the big number $N^{2}$, would be much larger than in the standard case. 


\section{Features of the 5D solution dual to a 4D evaporating black hole}

The analogy between (52) and (53) and between (56) and (54) is somewhat surprising. We shall now use it to conjecture some of the crucial features of the actual timedependent 5D solution allowing the holographic interpretations (52) and (56) in the dual theory. We stress that our considerations concern configurations where the mass is large, i.e. as long as $r_{H} \gg 1 / k$. Indeed, when $r_{H}$ becomes of order $1 / k$ it is not clear whether the holographic conjecture [7] still holds.

We recall that in braneworlds there are two different definitions of apparent horizons, the brane apparent horizon $r_{\text {brane }}^{\mathrm{AH}}$, defined with respect to photons which propagate along null geodesics of the 4D brane, and the bulk apparent horizon $r_{\text {bulk }}^{\mathrm{AH}}$, referring to gravitons which follow null geodesics of the full 5D spacetime. The static RS2 black string, for which $r_{\text {brane }}^{\mathrm{AH}}=r_{\mathrm{bulk}}^{\mathrm{AH}}=2 M$, is a special case. Indeed, in [22] it was numerically shown that in time-dependent braneworld black hole solutions brane and bulk horizons are generally distinct, the brane apparent horizon being always larger than the (brane projected) bulk horizon.

The black strings null geodesics (25) and (27), along with their associated surfaces $r=2 M$ and $r_{\text {bulk }}(z)$ in (48), (49), allowed us to reproduce such feature. It is thus tempting to speculate that $r_{\text {bulk }}(z)$ simulates the bulk apparent horizon $r_{\text {bulk }}^{\mathrm{AH}}$ of the actual $5 \mathrm{D}$ time dependent solution, its projection on the brane playing the role of the event horizon in the dual theory. In a way this is not too surprising, given that (classical) gravitons cannot escape from inside $r_{\text {bulk }}^{\mathrm{AH}}$ and, similarly, the dual (quantum) CFT modes cannot be emitted from the interior of $r^{\mathrm{EH}}{ }^{6}$

A large "quantum ergosphere" $r_{\text {bulk }}^{\mathrm{AH}}<r<r_{\text {brane }}^{\mathrm{AH}}$ would then form on the brane. The dual Hawking radiation thus suggests that 5D gravitational waves will be emitted from this region into the bulk, the typical wavelength of the emitted waves being of the order of $r_{\text {brane }}^{\mathrm{AH}}-r_{\text {bulk }}^{\mathrm{AH}} \sim \frac{1}{M k^{2}}{ }^{7}$ For large black holes we have that $\frac{1}{M k^{2}} \ll \frac{1}{k}$, and so the (local) energy of the KK modes is large, corresponding to the emission of "heavy" gravitons as in [24]. ${ }^{8}$ These modes correspond to the near-horizon 'transplanckian' CFT modes in the original derivation by Hawking. In our context, being the dual CFT cutoff at energies $\sim k$ it is not clear what they correspond to, since we would need to know its UV completion [27]. ${ }^{9}$

A suggestive possibility is the one envisaged in figure 2, bulk massive modes emitted

\footnotetext{
${ }^{6}$ Probably a careful analysis based on the full solution will rather identify the brane projection of the bulk event horizon $\left(\geq r_{\mathrm{bulk}}^{\mathrm{AH}}\right)$ with the dual event horizon $r^{\mathrm{EH}}\left(\leq r^{\mathrm{AH}}\right.$ in semiclassical evaporating spacetimes).

${ }^{7}$ Note that for large $A d S$ black holes, the quasinormal modes have wavelength $\sim \frac{1}{k^{2} r_{+}}$with $r_{+}$the horizon radius [23].

${ }^{8}$ Standard arguments [25] concerning the suppression of bulk radiation due to small function overlap between the localized brane black hole and the emitted bulk modes should not apply. We do not consider here effects due to rotation [26].

${ }^{9}$ Transplanckian effects are expected to be suppressed, for large black holes, by some positive power of $\frac{1}{M k}$. We thank R. Emparan and N. Kaloper for discussion on these points.
} 
from the region $r_{\text {bulk }}^{\mathrm{AH}}<r<r_{\text {brane }}^{\mathrm{AH}}$ will come back to the brane just above $r_{\mathrm{brane}}^{\mathrm{AH}}$. In the dual theory, the natural interpretation would be that of CFT modes tunneling through the horizon, as described in [28]. It was already suggested in [29] that gravitational waves traveling between two points on the brane through a null geodesics in the bulk can appear to travel faster than light. If so, from the brane point of view, this would justify the "leakage" of the dual CFT modes through the horizon. It remains to be seen whether such process really takes place or not in the actual time-dependent solution. ${ }^{10}$

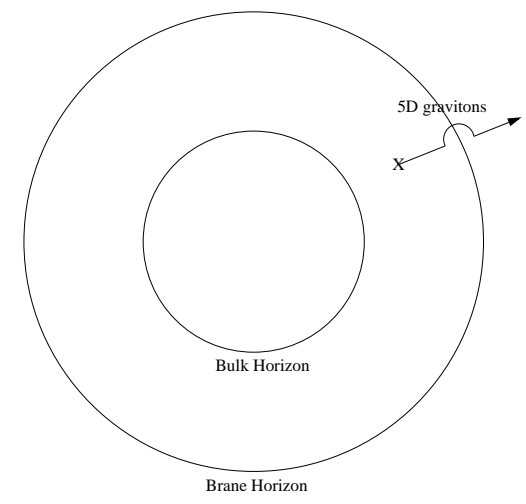

Figure 2: Dual of Hawking radiation as tunneling in braneworld.

Finally, we wish to show that for the full black string (23) the boundary effect described in this paper disappears. Indeed, in this case the dual CFT lives in the boundary at infinity of AdS with no gravity. This is achieved by letting the brane position $z_{\text {brane }} \rightarrow-\infty$. The boundary projection of our results (50) and (49) (or (48)) gives, for this case, $\frac{d r}{d v} \rightarrow 0$ and $r_{\text {bulk }} \rightarrow 2 M$ (i.e. no "evaporation" in the boundary theory and, consequently, no "horizon splitting" effect). This is closely related to our choice $C=0$ made in (47) to get (48). This is a qualitative difference with respect to the GL instability, which always holds irrespective of whether the brane is present or not.

\section{Conclusions}

To sum up briefly the results presented in this paper, holographic arguments applied to the propagation of bulk massive modes in the RS2 black string (5), namely our results (52) and (56), led us to conjecture some of the basic features of the actual time dependent solution describing an evaporating black hole on the brane, its horizon structure and the possible classical bulk dual of the tunneling mechanism for Hawking radiation (fig. 2).

\footnotetext{
${ }^{10}$ In a brane cosmological setting, gravitons leaving the brane to the bulk and then bouncing back to the brane have been shown to be present in the case of a bulk 5D Vaidya-AdS black hole in [30].
} 


\section{Acknowledgements}

G.P.P. wishes to thank Stephen Hawking for many useful discussions. We also thank Roberto Balbinot, David Langlois and José Navarro-Salas for interesting discussions. A. F. acknowledges the Spanish grant FIS2005-05736-C03-03 and the EU Network MRTN-CT-2004-005104 for financial support. G.P.P. is supported by PPARC and the Gates Cambridge Trust.

\section{References}

[1] J. M. Maldacena, Adv. Theor. Math. Phys. 2 (1998) 231 [Int. J. Theor. Phys. 38 (1999) 1113] [arXiv:hep-th/9711200].

O. Aharony, S. S. Gubser, J. M. Maldacena, H. Ooguri and Y. Oz, Phys. Rept. 323 (2000) 183 [arXiv:hep-th/9905111].

[2] L. Randall and R. Sundrum, Phys. Rev. Lett. 83 (1999) 4690 [arXiv:hep-th/9906064].

[3] J. Garriga and T. Tanaka, Phys. Rev. Lett. 84 (2000) 2778 [arXiv:hep-th/9911055].

[4] M. J. Duff, Phys. Rev. D 9 (1974) 1837.

[5] M. J. Duff and J. T. Liu, Class. Quant. Grav. 18 (2001) 3207 [Phys. Rev. Lett. 85 (2000) 2052] [arXiv:hep-th/0003237].

[6] P. R. Anderson, R. Balbinot and A. Fabbri, Phys. Rev. Lett. 94 (2005) 061301 [arXiv:hep-th/0410034].

[7] T. Tanaka, Prog. Theor. Phys. Suppl. 148 (2003) 307 [arXiv:gr-qc/0203082].

R. Emparan, A. Fabbri and N. Kaloper, JHEP 0208 (2002) 043 [arXiv:hep-th/0206155].

[8] R. Emparan, G. T. Horowitz and R. C. Myers, JHEP 0001 (2000) 007 [arXiv:hep-th/9911043].

R. Emparan, G. T. Horowitz and R. C. Myers, JHEP 0001 (2000) 021 [arXiv:hep-th/9912135].

[9] C. Germani and G. P. Procopio, Phys. Rev. D 74 (2006) 044012 [arXiv:hep-th/0605068].

[10] N. Dadhich, R. Maartens, P. Papadopoulos and V. Rezania, Phys. Lett. B 487 (2000) 1 [arXiv:hep-th/0003061].

N. Deruelle, arXiv:gr-qc/0111065.

P. Kanti and K. Tamvakis, Phys. Rev. D 65 (2002) 084010 [arXiv:hep-th/0110298].

R. Casadio, A. Fabbri and L. Mazzacurati, Phys. Rev. D 65 (2002) 084040 [arXiv:gr-qc/0111072]. 
C. Charmousis and R. Gregory, Class. Quant. Grav. 21 (2004) 527 [arXiv:gr-qc/0306069]

H. Kudoh, T. Tanaka and T. Nakamura, Phys. Rev. D 68 (2003) 024035 [arXiv:gr-qc/0301089].

H. Kudoh, Phys. Rev. D 69 (2004) 104019 [Erratum-ibid. D 70 (2004) 029901] [arXiv:hep-th/0401229].

G. Kofinas, E. Papantonopoulos and V. Zamarias, Phys. Rev. D 66, 104028 (2002) [arXiv:hep-th/0208207].

[11] A. Chamblin, S. W. Hawking and H. S. Reall, Phys. Rev. D 61 (2000) 065007 [arXiv:hep-th/9909205].

[12] A. L. Fitzpatrick, L. Randall and T. Wiseman, JHEP 0611 (2006) 033 [arXiv:hep-th/0608208].

[13] T. Tanaka, arXiv:gr-qc/0402068.

[14] R. Gregory and R. Laflamme, Phys. Rev. Lett. 70 (1993) 2837 [arXiv:hep-th/9301052].

R. Gregory and R. Laflamme, Nucl. Phys. B 428 (1994) 399 [arXiv:hep-th/9404071].

[15] R. Gregory, Class. Quant. Grav. 17, L125 (2000) [arXiv:hep-th/0004101].

[16] A. Chamblin and A. Karch, Phys. Rev. D 72, 066011 (2005) [arXiv:hep-th/0412017].

[17] J.M. Bardeen, Phys. Rev. Lett. 46 (1981), 382

[18] A. Fabbri and G. P. Procopio, arXiv:0705.3363 [gr-qc].

[19] S. W. Hawking, Commun. Math. Phys. 43 (1975) 199 [Erratum-ibid. 46 (1976) 206].

[20] J. W. York, in Quantum Theory of Gravity: Essays in Honor of the Sixtieth Birthday of Bryce S. DeWitt, edited by S. Christensen (Adam Hilger, Ltd., Bristol, 1984).

[21] J. W. York, Phys. Rev. D 28 (1983) 2929.

R. Balbinot, Phys. Rev. D 33 (1986) 1611.

[22] T. Shiromizu and M. Shibata, Phys. Rev. D $62 \quad$ (2000) 127502 [arXiv:hep-th/0007203].

[23] G. T. Horowitz and V. E. Hubeny, Phys. Rev. D 62 (2000) 024027 [arXiv:hep-th/9909056].

[24] D. Langlois, L. Sorbo and M. Rodriguez-Martinez, Phys. Rev. Lett. 89, 171301 (2002) [arXiv:hep-th/0206146]. 
[25] R. Emparan, G. T. Horowitz and R. C. Myers, Phys. Rev. Lett. 85, 499 (2000) [arXiv:hep-th/0003118].

[26] D. Stojkovic, Phys. Rev. Lett. 94, 011603 (2005) [arXiv:hep-ph/0409124].

[27] N. Arkani-Hamed, M. Porrati and L. Randall, JHEP 0108, 017 (2001) [arXiv:hep-th/0012148].

[28] S. Massar and R. Parentani, Nucl. Phys. B 575 (2000) 333 [arXiv:gr-qc/9903027]. M. K. Parikh and F. Wilczek, Phys. Rev. Lett. 85 (2000) 5042 [arXiv:hep-th/9907001].

V. A. Berezin, A. Boyarsky and A. Y. Neronov, Grav. Cosmol. 5, 16 (1999) [arXiv:gr-qc/0605099].

[29] C. Csaki, J. Erlich and C. Grojean, Nucl. Phys. B 604, 312 (2001) [arXiv:hep-th/0012143].

C. Csaki, J. Erlich and C. Grojean, Gen. Rel. Grav. 33, 1921 (2001) [arXiv:gr-qc/0105114].

[30] D. Langlois and L. Sorbo, Phys. Rev. D 68, 084006 (2003) [arXiv:hep-th/0306281]. 